\title{
Two kinds of exploratory models
}

\begin{abstract}
Michela Massimi
I analyse the exploratory function of two main modelling practices: targetless fictional models and hypothetical perspectival models. In both cases, I argue, modelers invite us to imagine or conceive something about the target system, which is either known to be non-existent (fictional models) or just hypothetical (in perspectival models). I clarify the kind of imagining or conceiving involved in each modelling practice, and I show how each-in its own right-delivers important modal knowledge. I illustrate these two kinds of exploratory models with Maxwell's ether model and SUSY models at the LHC.
\end{abstract}

\section{Introduction}

Much of the literature on scientific modelling has concentrated on an important cluster of inter-related topics concerning what a model is, and how models represent, just to mention two examples. Yet there is an important function of scientific modelling that has received far less attention in the philosophical literature until recently: their exploratory function (see Gelfert 2016 for a recent re-appraisal). ${ }^{1}$

The exploratory function of scientific models has traditionally been regarded as part and parcel of their representational function. On the received view, models fulfil their exploratory function by offering abstract and idealized representations of their target systems. In this paper, I argue that there is more to the exploratory function of scientific models than the received view has suggested so far. Some scientific models fulfil their exploratory function not necessarily by offering idealised yet accurate and veridical representations of the target system. In important contexts and circumstances, some scientific models fulfil their exploratory function by inviting us instead to imagine or conceive non-veridical scenarios about the target system so as to deliver modal knowledge about what is possible. Two modelling practices are exemplary in this respect. Following Michael Weisberg's (2013) terminology, let us label them as follows:

\footnotetext{
${ }^{1}$ Gelfert (2016, 83-97) describes exploratory models as fulfilling four distinct (not exhaustive) functions: (1) starting point for future inquiry; (2) they feature in proof-of-principle demonstrations; (3) they generate potential explanation of observed (type of) phenomena; (4) or lead to assessments of the suitability of the target. In this paper I'd like to add two further functions to this list: (5) delivering knowledge of causal possibilities; and (6) delivering knowledge of objective possibilities for hypothetical entities.
} 
Forthcoming in Philosophy of Science. Proceedings of the PSA 2018, Seattle.

(1) Targetless modeling: scientific models without a target system. Within this practice, fictional models, i.e. models of knowingly false and non-existent entities (along the lines of Bokulich 2009, 2016) ${ }^{2}$ are particularly interesting.

(2) Hypothetical modeling: scientific models whose target system is hypothetical. Within this practice, perspectival models are an important family of incompatible (or even inconsistent) models for the same target system (along the lines of Giere 2006, Rueger 2005, Morrison 2011, and Massimi 2018). ${ }^{3}$

What good are targetless and hypothetical models when it comes to explore what might be the case in nature? How can genuine modal knowledge (about how things might be in nature) be obtained by conceiving non-veridical scenarios in targetless and hypothetical models? The goal of this paper is to get clear about the procedures through which these two families of models, respectively, deliver important kinds of modal knowledge. As such, my goal is neither to advocate (or criticize) fictionalism about models, nor advocate (or criticize) the use of explanatory falsehoods in modelling. ${ }^{4}$ Instead, my goal is to clarify how targetless and hypothetical models successfully deliver varieties of modal knowledge in science, thanks to their specific kind of imagining or conceiving non-veridical scenarios. Thinking of what goes on in scientific modelling along the lines of some kind of imagining, or, to be more precise, physically conceiving something about the target system is key to clarifying how models deliver important modal knowledge in science, notwithstanding false posits, fictional targets, or purely hypothetical entities they postulate.

\footnotetext{
${ }^{2}$ Frigg (2010) distinguishes two meanings for fictional models: (1) fiction as imagination; and (2) fiction as falsity, or nonexistence. Frigg himself endorses (1). Other authors (e.g. Bokulich) rely on (2), which is also how I am going to use the term here. Some philosophers of science have taken the highly abstract and idealized nature of the representation afforded by models as the sign that fictionalism is the correct ontology of scientific modeling (no matter whether its target system is real, or hypothetical, or simply non-existent) qua make-believe games along Kendall Walton's theory. In what follows, I am not interested in fictionalism as an ontology of modeling. I am interested instead in what kind of modal knowledge scientists can obtain from models whose target system is known to be false and non-existent, i.e. I use fiction as in (2).

3 The class of "perspectival models" as inconsistent models for the same target system spans across both hypothetical models and concrete models. Different models for the atomic nucleus (quark models, cluster models, shell model, liquid drop models) can be regarded as perspectival models for a target system known to exist and be real (the nucleus) — see Morrison (2011). Different models for supersymmetric particles (my example in what follows) can be regarded as perspectival models for a hypothetical target system (i.e., supersymmetric particles that have been hypothesised but not yet discovered as of today).

${ }^{4}$ Some philosophers of science have taken the highly abstract and idealized nature of scientific modeling as a springboard for re-evaluating the explanatory importance of falsehoods in science (e.g., Bokulich 2009, 2016). Others have taken the ubiquitous use of false-yet-explanatory models as the sign that the aim of science is to offer non-factive understanding, rather than truth (see Elgin 2017; Potochnik 2017). My focus in what follows is on particular varieties of modal knowledge delivered by two identified classes of scientific models (rather than on the topic of scientific understanding or explanatory falsehood as such). Hence my analysis should be read as orthogonal to the latter debate, and at most as expanding and complementing the existent literature on the topic.
} 
Section 2 introduces the notion of "physical conceivability" to describe the kind of imagining that goes on in targetless and hypothetical modelling. I analyse how fictional models-in the meaning just clarified-resort to a particular version of "physical conceivability" about the non-existent target. I illustrate this point with Maxwell's ether model where the fictional model acts as a proxy for facilitating the acquisition of a particular kind of modal knowledge (i.e. knowledge of what is causally possible). This is done-I contend-by ascribing essential properties and relations to the phenomenon under study via law-driven analogical reasoning with concrete models in other domains.

Section 3 discusses hypothetical perspectival models, well exemplified by a plurality of models for supersymmetric particles in high-energy physics. This modelling practice deploys a slightly different notion of physical conceivability. Its purpose is to deliver modal knowledge about what might be objectively (rather than causally) possible about the hypothetical target system. In this case, the modal gap is filled via subjunctive conditionals that feature in bootstrapped inferences, without any essential-property ascription.

\section{How targetless fictional models act as a guide to what is causally possible}

That fictional models can be a guide to what is causally possible is not a foregone conclusion. For how can a model of a knowingly false (or fictional) target system be expected to deliver knowledge at all? In this Section I argue that fictional models can in fact act as a guide to knowledge of causal possibilities in interesting and unexpected ways. Targetless fictional models, I maintain, invite scientists to physically conceive non-veridical scenarios about a non-existent target system. The goal is to allow scientists to explore possible causal mechanisms behind a given phenomenon of interest, for which the non-existent fictional target provides a proxy. But what is physical conceivability, to start with?

Physical conceivability is a kind of epistemic conceivability (along the lines of Yablo 1993) that is consistent with known laws of nature. Here is a first stab at a definition:

$p$ is physically conceivable for an epistemic subject S (or an epistemic community C) if S's (or C's) imagining that $p$ not only complies with the state of knowledge and conceptual resources of S (or C) but it is also consistent with the laws of nature known by $\mathrm{S}$ (or C).

In targetless fictional models, scientists often conceive properties and relations in a given nonexistent target system. Their goal is to identify a possible causal mechanism behind a given phenomenon that the fictional target system is a proxy for. This conceivability exercise is driven 
by laws of nature in that some of the properties and relations attributed to the fictional target system mimic analogically properties and relations that—according to known laws of natureare typically attributed to non-fictional, concrete target systems that serve as template for the fictional models.

Consider, for example, Maxwell's honeycomb model of the ether, which assumed an ether with hexagonal vortices that by rotating generated a magnetic field (whose strength was assumed to be proportional to the angular velocity of the vortices). Maxwell's (1861/2) model is a targetless fictional model. Maxwell referred to it as a "collection of imaginary properties" and not even a hypothetical fluid. Maxwell's honeycomb model of the ether delivered modal knowledge about what is causally possible in the phenomenon of electromagnetic induction. Despite being the model of a non-existent object (the ether)—and hence involving non-veridical representations- the honeycomb model was a guide to knowledge of causal possibilities obtained through analogy with concrete models in hydrodynamics.

In the late 1820s and early 1830s, Michael Faraday made important discoveries about the phenomenon of electromagnetic induction, following up on the early work of Hans Christian Ørsted. Electromagnetic induction is the surprising phenomenon whereby the passage of electricity in a wire is able to twist sideways a nearby magnetic needle (as first observed by Ørsted), as much as the movement of a magnet inside a metal ring wrapped with electric wire induces a displacement of electric current inside the wire (as Faraday first recorded it). These experiments offered the first evidence that an important connection existed between electricity and magnetism. Faraday was able to formulate a law for electromagnetic induction, which related the electricity induced in the wire (or better its induced voltage) to the rate of change over time of the magnetic field (conceived by Faraday as magnetic lines of force, or magnetic flux around the coil). But what was the causal mechanism responsible for electromagnetic induction? How can magnetic lines of force induce electricity in a nearby wire?

Throughout the nineteenth century scientists came up with elaborated models of the ether in the hope to answer this question. Among them, James Clerk Maxwell, thirty years after Faraday's discovery, built his ether model based on an analogy with hydrodynamics. Maxwell was aware of Helmholtz's (1858) work on hydrodynamics and vortex fluid and came up with an intentionally fictional honeycomb model of the ether, whereby Faraday's lines of magnetic force could be thought of as analogous with lines of fluid motion; and electric displacement as analogous with rotation in fluid (see Bokulich 2015 for a historical discussion). Maxwell's fictional model, I contend, delivered modal knowledge about how the magnetic field might cause electric displacement in the wire by imagining that there was a specific relation between the strength of the magnetic 
field (or better, its rate of change over time) and the displacement of electric current (or better, the induced voltage in the wire).

This is an exercise in physical conceivability; or better, in a first variety of it (let us call it "physical conceivability $\mathrm{LD}_{\mathrm{LD}}$ ") that can be better qualified as law-driven (LD) conceivability. Laws of nature-in this case, Faraday's law of electromagnetic induction but also Helmholtz's equations for fluid dynamics — drove the analogical reasoning with fluid dynamics upon which Maxwell patterned his ether model. This law-driven exercise of imagining perfectly spherical cells moving among hexagonal cylinders in an elastic ether-in analogy with water vortices-led Maxwell to his breakthrough. Namely, the discovery that the elasticity of the medium for electromagnetic induction (magnetic ether) was one and the same as the elasticity of the medium for the transmission of light (luminiferous ether). ${ }^{5}$ From there, the step to the conclusion that light was, after all, nothing but an electromagnetic wave was a short one. In the following decade leading to his Treatise on electricity and magnetism in 1873, Maxwell abandoned the model of the ether and codified the equations for the electromagnetic field that bear his name. The honeycomb model of the ether proved a crucial stepping-stone in this breakthrough.

Maxwell's model illustrates the way in which targetless fictional models by and large act as a guide to knowledge of causal possibilities via analogical reasoning with concrete models in other fields (be it Fourier's treatment of heat flow behind Lord Kelvin's vortex sponge ether model; or Helmholtz's fluid dynamics behind Maxwell's honeycomb model). Analogical reasoning allows targetless fictional models to attribute properties to an otherwise purely imaginary and non-existent target system (ether), in analogy with the property attribution that goes on in concrete models (say, hydrodynamic models). Fictional models ultimately deliver important modal knowledge about what is causally possible concerning a phenomenon under study (e.g. electromagnetic induction), by attributing to a fictional entity some essential properties and relations $P_{1} \ldots P_{n}, R_{1 \ldots} R_{j}{ }^{6}$ which via suitably keying are translated into essential properties and relations $\mathrm{Q}_{1} \ldots \mathrm{Q}_{\mathrm{m}}, \mathrm{T}_{1} \ldots \mathrm{T}_{\mathrm{s}}{ }^{7}$ imputed to the phenomenon. ${ }^{8}$

\footnotetext{
5 This is how Maxwell (1861/2, pp. 13-14 and 21) presented his model: "we may conceive that the electricity in each molecule is so displaced that one side is rendered positively, and the other negatively electrical, but that the electricity remains entirely connected with the molecules ...I have deduced from this result ...that the elasticity of the magnetic medium in air is the same as that of the luminiferous medium, if these two coexistent, coextensive, and equally elastic media are not rather one medium.... we can scarcely avoid the inference that light consists in the transverse undulations of the same medium which is the cause of electric and magnetic phenomena."

${ }^{6}$ For example, the property of being made of adjacent hexagonal cylinders, and the property of having spherical cells as idle wheels to mitigate friction among cylinders; but also the relation between the rotation of the hexagons and the ensuing motion of the spherical cells in between them.

${ }^{7}$ For example, the strength of the magnetic field and the direct proportionality between the strength of the magnetic field and the induced electric displacement in the wire.

8 This characterization is an illustration of Frigg and Nguyen's (2016) so-called DEKI account of scientific
} 
By physically conceiving LD $_{\text {of }}$ an ether with the essential properties of hexagonal cylinders and idle wheels, Maxwell's fictional model could explore the possible causal mechanism for electromagnetic induction. The model did not identify the ether as the causal mechanism. For the model was not meant to be an accurate or veridical representation of any actual or even possible state of affairs in nature. Instead, the model allowed to explore the phenomenon of electromagnetic induction by ascribing some essential properties to a fictional target that could be used as a proxy to understand how for example a magnetic field might causally induce electric current in a wire (e.g. how strong the magnetic field would have to be for electric displacement to be generated; whether the electric displacement would depend on the nature of the dielectric; and so on). The exploratory nature of Maxwell's model consisted in its ability to deliver useful modal knowledge about causal possibilities through an exercise of physically conceiving LD $_{\text {a knowingly }}$ non-existent entity (ether) as having some essential properties and relations, and see how far the analogy with water's vortex motion could lead. This is modal knowledge gained via analogical reasoning with concrete models.

But this exercise has also its glaring limits. Analogical reasoning with concrete models can lead to misleading essential property attribution. For example, the fictional ether got ascribed some classical quantities (e.g. momentum) in analogy with fluid motion, as if momentum were a kinematic property of structural bearers inside the ether (be it vortices, gyroscopes or else) rather than a dynamical property of the electromagnetic field. This misleading essential property attribution led to the failure of an entire tradition of mechanical models of the ether in the nineteenth century; and it reveals the shaky foundations on which this exercise in physical conceivability $\mathrm{LD}_{\mathrm{D}}$ rested. Taking heat flow or fluid flow as an analogue upon which to model property ascription to the electromagnetic field had its limits, as the history of ether models in the nineteenth century shows. Nonetheless, the physical conceivabilityLD afforded by ether models turned out to be crucial in the exploratory process that led to the development of electromagnetic theory.

\section{How hypothetical perspectival models act as a guide to what is objectively possible}

representation as an account that is meant to encompass scientific representation in all sorts of models (not just targetless fictional ones). As it will become clear in the next Section, other kinds of models may resort to different representational strategies. 
And it is not just targetless fictional models that deliver important modal knowledge in science. Hypothetical perspectival models prove equally pivotal to exploring what might be the case in nature. This is well exemplified by the bewildering plurality of hypothetical models currently employed by particle physicists to search for supersymmetric particles. Long theorized to fill some gaps in the current Standard Models (e.g. the naturalness problem, among others), supersymmetric particles (SUSY) have not been found as of today. In this Section, I briefly present one influential modeling practice to search for SUSY at the Large Hadron Collider (CERN). This modelling practice, I argue, deploys a slightly different notion of physical conceivability qua law-bounded (LB) conceivability for the purpose of delivering modal knowledge about what I call objective possibilities (possibilities concerning the very existence of the model's hypothetical target system). Physical conceivability ${ }_{\mathrm{LB}}$ is an exercise in conceiving or imagining something (e.g. properties or behaviour) about the hypothetical entity with the goal of facilitating inferences about its very existence (rather than identifying causal mechanisms for phenomena that the target system is a proxy for). Here laws of nature do not directly drive the conceiving or the analogical reasoning behind it. Laws of nature fix instead the nomological boundaries within which inferences about the hypothetical target system can take place. As a result, physical conceivability $\mathrm{LB}_{\mathrm{L}}$ acts as a guide to a different kind of modal knowledge in science as knowledge of objective possibilities (i.e. knowledge of what is objectively possible in nature).

This is exemplarily illustrated by models of supersymmetric particles at the LHC. One of the most difficult tasks for the particle physics community at the LHC is to devise modelling techniques that by conceiving of hypothetical SUSY particles as being thus-and-so (within broad nomological constraints) can provide an effective method for hunting them (assuming they exist). The modelling techniques in question are an example of hypothetical perspectival models (see Massimi 2018 on perspectival modeling): i.e. models that play with physically conceivable $\mathrm{L}_{\mathrm{LB}}$ scenarios about a putative entity $z$ so as to allow scientists to infer some of the very many (and inconsistent) possible ways in which z.might exist in nature. One of such modelling techniques takes the name of phenomenological Minimal SuperSymmetric Model (or pMSSM-19, see ATLAS 2015 and Massimi 2018 for a brief discussion). The task of pMSSM-19 is to physically conceive $e_{L B}$ a plurality of SUSY scenarios within broad nomological constraints (i.e. R parity conservation and consistent electroweak symmetry breaking, among others).

Each of these different scenarios (or, to use a more precise terminology, "model points") portrays SUSY particles that might have inconsistent mass values assignment and inconsistent decay modes. Each model point assigns different numerical values to the 19 parameters so that the same hypothetical SUSY particle (e.g. a Higgsino) might end up having a mass of $2800 \mathrm{GeV}$ 
in a model point, and a mass of $4000 \mathrm{GeV}$ in another model point (and different decay modes too). Out of the 500 million model points originally conceivable, the ATLAS Collaboration (2015) initially sampled 310,327 model points, which were randomly selected. Physicists do not even know whether there are SUSY particles after all, and no insight of putative essential properties is intended to be delivered by the pMSSM-19, whose task is not to offer an accurate or veridical representation of what there might be, but instead to trim down the bewildering space of 500 million conceivable model points to a more manageable size. Thus, here we have another interesting example of how exploratory models are a vehicle to modal knowledge without necessarily offering an accurate or veridical representation.

The pMSSM-19 has a primarily exploratory function in that it allows physicists to randomly select a large number of model points with the hope that-by sampling a sufficiently high number of them-SUSY particles (were they to exist) could be hunted. The pMSSM-19 help physicists to model a number of physically conceivable $e_{L B}$ scenarios (within broad laws of nature so that theoretically pathological scenarios get discarded immediately). The task then becomes how to rule out (with 95\% confidence level) large portions of these physically conceivable $_{\mathrm{LB}}$ scenarios using experimental evidence coming from proton-proton collisions at the LHC. SUSY perspectival models do not ascribe essential properties to hypothetical entities.? Instead they model the many possible (and inconsistent) ways SUSY particles could be produced, so as to exclude those not supported by experimental data.

SUSY pMSSM-19 perspectival models fill the gap between physical conceivability ${ }_{\mathrm{LB}}$ and objective possibilities then not via essential-property ascription mediated by analogical reasoning (as with targetless fictional models). But instead, by delivering a particular type of howpossible inferences: How could $x_{1}, \ldots x_{z}$ be possible, were $y_{1}, \ldots y_{n}$ physically conceived $d_{L B}$ thus-andso? For example, how could the Higgsino decay, were the model point 18898934 fine-tuned in this way? Any conceivable model point that has not been ruled out (at $95 \%$ confidence level) by experimental evidence coming from proton-proton collisions at LHC remains a live objective possibility.

\footnotetext{
${ }^{9}$ Let us be clear again on this point. One might retort that surely any SUSY model is bound to ascribe some essential properties to these hypothetical entities. For example, a model point 10407816 (fine-tuned 57) might be said to ascribe to the hypothetical SUSY particle called Higgsino the property of having mass $4000 \mathrm{GeV}$. This objection, however, misses the main point here. Namely, it is not the primary task of pMSSM-19 model points to ascribe essential properties to these hypothetical entities (which for all we know as of today might well turn out to be non-existent). Instead their task is to model the possible ways in which these putative entities might exist (within broad theoretical and experimental constraints). Most importantly, such modelling is not downstream of any prior knowledge of essential properties (precisely because physicists as of today do not even know whether these putative entities exist or not; a fortiori they are not inferring their possible ways of existing from any prior essential-property-ascription).
} 
But here is a worry. How can SUSY perspectival models reliably track what there might exist? Is not there a genuine risk of treading water in the conceivability exercise given the hypothetical nature of the target system? (as indeed some physicists feel about SUSY models and the lack of progress in finding evidence for them as of today). This worry might be deflated. Consider the distinctive bootstrapping procedure through which new pieces of experimental evidence enter in the how-possible inferences to provide a firm anchor for what might be the case (under antecedently assumed physically conceivable LB $_{\text {s }}$ scenarios). As new pieces of experimental evidence $e_{1} \ldots e_{3}$ are brought in, they refine the how-possible inferences by excluding more and more of the live objective possibilities $x_{1}, \ldots x_{\mathrm{z}}$. This is because new evidence gradually and increasingly constrains what is physically conceivable $\mathrm{LB}_{\text {B }}$ (i.e. the antecedents $y_{1}, \ldots y_{n}$ of the howpossible inference). ${ }^{10} \mathrm{Via}$ bootstrap, more and more stringent how-possible inferences are obtained so that more and more possible SUSY particles get excluded with high confidence level as new energy regions at LHC are probed:

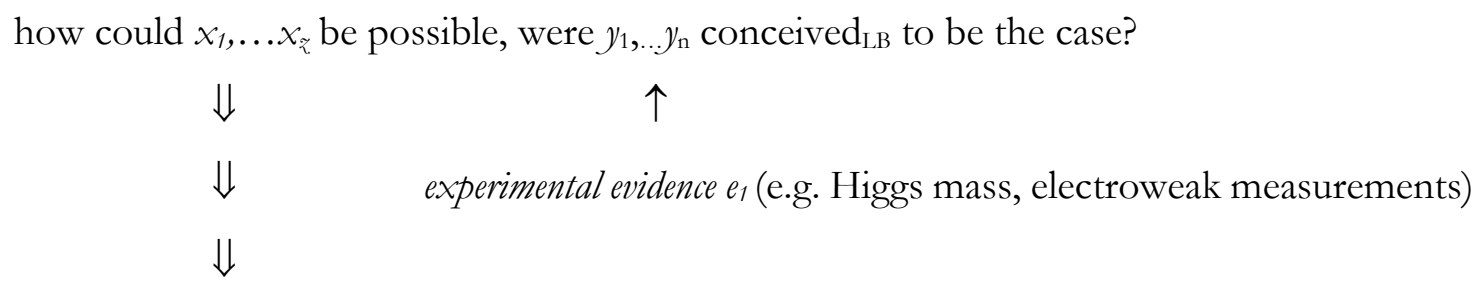

how could $x_{1}, \ldots x_{z-1}$ be possible, were $y_{1}, \ldots y_{\mathrm{n}-1}$ conceived $d_{\mathrm{LB}}$ to be the case?

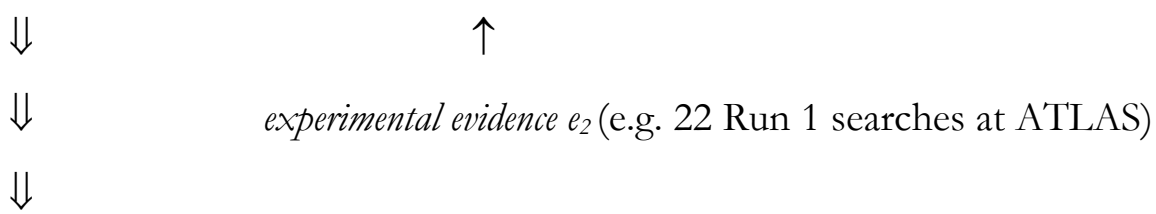

how could $x_{1}, \ldots x_{z-2}$ be possible, were $y_{1}, \ldots y_{\mathrm{n}-2}$ conceived $\mathrm{L}_{\mathrm{LB}}$ to be the case?

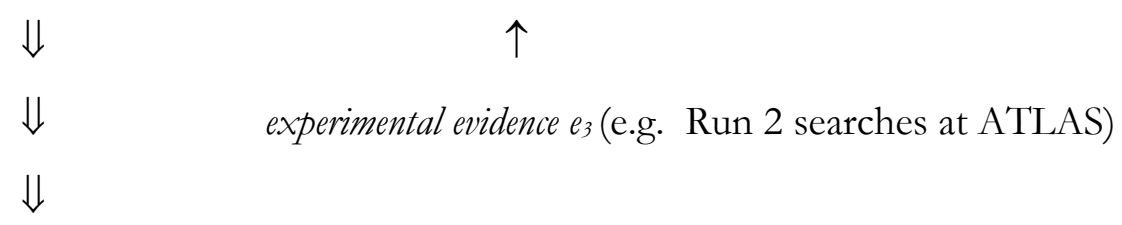

how could $x_{1}, \ldots x_{z-3}$ be possible, were $y_{1}, \ldots y_{\mathrm{n}-3}$ conceived $_{\mathrm{LB}}$ to be the case?

To illustrate the bootstrap at work in these how-possible inferences, let us go back to our example. The ATLAS Collaboration (2015) used the pMSSM-19, with minimal nomological constraints (R-parity exactly conserved, among others). The resulting 500 million model points

\footnotetext{
10 To simplify the matter, in the scheme above I assumed that only one physically conceivable $\mathrm{LB}_{\mathrm{B}}$ state is ruled out in the antecedent at any round of new experimental evidence. But in practice, obviously, very many physically conceivable $\mathrm{LB}_{\mathrm{LB}}$ scenarios are ruled out at any one time.
} 
were indirectly constrained using experimental evidence coming from the measurement of the Higgs mass, electroweak measurement, and heavy flavor physics among others. Only 310,327 model points survived this first round. And they were tested against the 22 Run1 searches, which trimmed them further down to $183,800 \mathrm{ca}$. model points. These surviving 183,800 model points featured objectively possible SUSY particles that were in turn the object of further comparison with Run 2 searches at higher $13 \mathrm{TeV}$ energies (analysis included 2-6 jets, 7-10 jets, multi-b, monojet and others). The outcome (Barr and Liu 2017) was that 15.7\% of the model points surviving Run 1 were excluded by early searches in Run 2 as Figures 1 and 2 show (with the hatched grey area in Fig. 1 indicating the excluded region from Run 1 at $8 \mathrm{TeV}$ for a hypothetical gluino-neutralino process, and the black area around it indicating the new exclusion region from Run 2 at $13 \mathrm{TeV}$ for the same process).

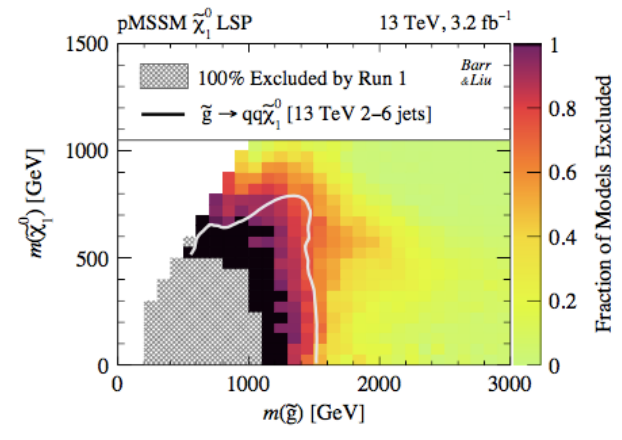

(a) Gluino-LSP plane.

Fig. 1

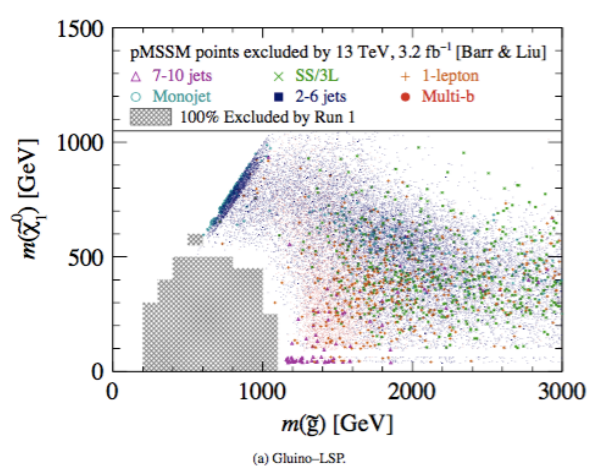

Fig. 2

Fig. 1 shows the fraction of models excluded by Run-2 for a possible decay of a light gluino into the lightest supersymmetric particle (LSP) in the space of possible pMSSM-19 models points, where the hatched grey area has already been excluded by Run 1 at ATLAS. Copyright: Barr and Liu 2017 (Fig. 11a). Reproduced under Open Access CC BY 4.0.

Fig. 2 shows in different colors the search technique (i.e. 2-6 jets, 7-10 jets, multi-b searches, and so forth) used to exclude the modal space of possible gluino-LSP processes at Run 2. Copyright: Barr and Liu 2017 (Fig. 1a). Reproduced under Open Access CC BY 4.0.

Further Run 2 analyses from proton-proton collisions at LHC are likely to trim down the number of possible surviving model points even further. And the question that looms large for the future 
of LHC is how to go about probing higher-energy regions, which are currently not accessible within the LHC energy regimes.

Scientists might have very good theoretical reasons for thinking that SUSY particles exist (i.e. naturalness problem, hierarchy problem, among others). They might have very sophisticated modelling practices for exploring what might be objectively possible. But ultimately, it is only incoming new experimental evidence that constrains and bootstrappingly refines the howpossible inferences so as to make more and more educated guesses on which SUSY particles (if any) there might be.

\section{Conclusion}

The overarching goal of this paper was to get clear on the exploratory function of two main classes of models: targetless fictional models and hypothetical perspectival models. In both these modelling practices, the exploratory function consists in imaging or physically conceiving non-veridical scenarios about a target system which is either known to be non-existent or just hypothetical. The upshot of this exercise is to be able to explore what is either causally or objectively possible. And the procedures through which fictional models and perspectival models respectively deliver kinds of modal knowledge is either via property ascription to a fictional target (acting as a proxy for a phenomenon of interest) or via bootstrapped howpossible inferences that get refined as more evidence becomes available. In either case, this is yet another powerful illustration of how models are able to carve out the space of what is possible, notwithstanding their false or hypothetical posits.

\section{Acknowlewdgments}

Earlier versions of this article were presented at the IHPST in Toronto, and at the Models and Simulations 8 conference in South Carolina. I thank the audiences, especially Jossi Berkovitz, Alisa Bokulich, Chris Pincock, Stephen Downes, Kevin Elliott, Axel Gelfert, Michael Miller, James Nguyen, Peter Mättig, Elay Schech, Michael Stoeltzner, Morgan Thompson, and Michael Weisberg for helpful comments. This article is part of a project that has received funding from the European Research Council under the European Union's Horizon 2020 research and innovation program (European Consolidator grant H2020-ERC-2014-CoG 647272, Perspectival Realism: Science, Knowledge, and Truth from a Human V antage Point). 


\section{References}

Barr, A. and J. Liu. 2017. "Analysing parameter space correlations of recent $13 \mathrm{TeV}$ gluino and squark searches in the pMSSM”. The European Physical Journal C 77: 202. Pp. 1-25.

Bokulich, A. 2009. "Explanatory Fictions". In Fictions in Science: Philosophical Essays on Modeling and Idealization, ed. M. Suarez, 91-109. New York: Routledge.

2015. "Maxwell, Helmholtz, and the Unreasonable Effectiveness of the Method of Physical Analogy". Studies in the History and Philosophy of Science 50: 28-37.

2016. "Fiction as a Vehicle for Truth: Moving Beyond the Ontic Conception". The Monist 99: 260-279.

Elgin, C. Z. 2017. True Enough. Cambridge: MIT Press.

Frigg, R. 2010. "Fiction in science". In Fictions and Models: New Essays, edited by J. Woods, Munich: Philosophia Verlag, 247-287.

Frigg, R., and J. Nguyen. 2016. “The Fiction View of Models Reloaded.” The Monist 99: 225-42.

Gelfert, A. 2016. How to do science with models. Dordrecht: Springer.

Giere, R. 2006. Scientific Perspectivism. Chicago: University of Chicago Press.

Massimi, M. 2018. "Perspectival modelling”. Philosophy of Science 85: 335-359.

Maxwell, J. C. 1861/2. "On Physical Lines of Force". Reprinted in (1890) The scientific papers of James Clerk. Maxwell. Dover: New York, 451-513.

Morrison, M. 2011. "One phenomenon, many models: inconsistency and complementarity". Studies in History and Philosophy of Science 42: 342-351.

Potochnik, A. 2017. Idealization and the aims of science. Chicago: University of Chicago Press.

Rueger, A. 2005. "Perspectival models and theory unification". British Journal for the Philosophy of Science 56: 579-594.

Weisberg, M. 2013. Simulation and similarity. New York: Oxford University Press.

Yablo, S. 1993. "Is conceivability a guide to possibility?". Philosophy and Phenomenological Research 53: $1-42$. 